MATEC Web of Conferences 44, 02090 (2016)

DOI: $10.1051 /$ matecconf $/ 20164402090$

(C) Owned by the authors, published by EDP Sciences, 2016

\title{
Ad hoc on-demand Distance Vector Routing Protocol Based on Load Balance
}

\author{
Yun Jie Zhu' ${ }^{1}$, Yong Qiang $\mathrm{Li}^{1}$, Qing Gang Fan ${ }^{1}$, Zhong Wang ${ }^{1}$ \\ ${ }^{1}$ Computer Staff Room,Xian Research Institute of High Technology, Xian 721006, China
}

\begin{abstract}
The ad hoc on-demand distance vector routing protocol based on load balance (AODVLB) is proposed aiming at the jitter problem. The turbulence phenomena in on-demand route protocol is presented, the delay forwarding technology is introduced to control the route building, a load calculation function have been designed. The results of simulation using ns 2 show that the method decreases $20 \%$ than AODV in jitter time, improves the networks' performance significantly.

Key Words. MANET, load balance, turbulent flow, jitter, AODV
\end{abstract}

\section{1、 introduction}

A mobile ad hoc network consists of wireless mobile nodes that can communicate with other nodes through wireless links without any fixed infrastructure. Mobile Ad Hoc network ${ }^{[1]}$ is a system of wireless mobile nodes which self-organize in dynamic and temporary network topologies. Ad hoc networks are easier to organize than wired networks and used in many applications, such as virtual classrooms, military communications, emergency search, rescue operations and data acquisition in hostile.

Jitter is a important performance on wireless networks.It reflects the delay variance in data transmission speed. In ensuring Quality of Service (QoS) provisioning, a network is expected to guarantee a set of measurable pre-specified service attributes to the users in terms of end-to-end performance, the lifetime of the networks, the packets loss rate and so on ${ }^{[2][3]}$ Recent Qos solutions are planned to operate on trusted environments and totally assume the participating nodes to be cooperative and well behaved. ${ }^{[4][5]}$

The major drawback of conventional on-demand routing protocols such as ad hoc on-demand distance vector protocol (AODV) is the absence of the QoS provision and consideration about load of nodes before routing. In this paper, we propose the enhanced ad hoc on-demand distance vector routing protocol which is modified to improve the networks jitter in mobile ad hoc networks. The AODVLB protocol involves the delay calculating function which can determine the delay from each node's load .When the nodes forward the RREQ packets, it helps in building a path that does not have nodes exhausted and selects a better path. It tries to establish a balance of load among the nodes.

\section{2、 Related Work}

The problem of trying to decrease the delay time of networks in MANET is not new. A number of previous works has addressed different strategies spanning different net layers.

The technique, constraining the scales of the subsets of forwarding nodes, is to reduce the cost of disseminating information by limiting the cardinality of the subset of nodes which retransmit a packet. The technique, optimizing the selecting path criterions, takes the delay、 hops into the path selecting metrics. The key point of these algorithms is the designation of path assessment function aiming the different requirements. It can be classified into multi-objective optimization in math. 
Article[6]presents the m-limited forwarding technique. In case of flooding, the number of messages increases geometrically with the distance from the source to the destination, while for $\mathrm{m}$-limited forwarding the increase is only linear. It gives a forwarding fitness function which determined weather does the node forward packets. In reality it reduces the power consumption as well as the waste of the network band-width, but the defect is increasing the computational complexity, in addition, the parameter of the forwarding fitness function is difficult for determining and need an amount of experiments to be modified.

When the destination gets the first RREQ, it waits for $\sigma \mathrm{t}$ time and collects all other RREQ coming in this time interval ${ }^{[7]}$. After $\sigma \mathrm{t}$ time, it calls the optimization function to determine the best path to select and send RREP. The Optimization function involves the time taken, the load and the hop count in determining the path that will give more reliable results.

Article [8] proposed a path building controlling mechanism. It take the hops, the load value into the RREQ packets and could improve the route generating, but the threshold is difficult to set.

\section{The AODVLB Protocol}

\subsection{Basic Theory}

A node disseminates a RREQ when it needs a route to a destination and does not have one available. When a node receives a RREQ, it first checks whether it has received a RREQ with the same Originator IP Address and RREQ ID within the last PATH_DISCOVERY_TIME. If such a RREQ has been received, the node silently discards the newly received RREQ. If a node does not generate a RREP then it will forwarding the RREQ. The destination node will generate the RREP based on some principles and send it out. ${ }^{[9][10]}$

\subsection{Algorithm Flow}

We proposed the AODVLB: Ad hoc on-demand Distance Vector routing Protocol Based on Load Balance. Its theory is as the same of the standard AODV, the difference is the usage of delay forwarding technology mentioned in the forth section in forwarding packets.
When a source node wants to transmit data to the destination node, first it checks whether there is any existing valid path in the routing table. If it exists, the node uses that path, otherwise, it sends RREQ to its neighbor nodes. When a node (either destination or intermediate) receives RREQ, it ensures that the received RREQ is not a duplicate RREQ, in order to prevent looping paths. If the neighbor node is the destination, it sends RREP. Otherwise, it waits for a delay and sends new RREQ to all their neighbor nodes to find the destination. When the destination gets the first RREQ, it waits for ot time and collects all other RREQ coming in this interval. After $\sigma$ time, it calls the optimization function to determine the best path to select and send RREP. It also stores some other relatively inferior paths as backup paths, which may be used if there is some network failure, thereby avoiding energy and time wastage in recalculating the path. When the source node gets the RREP, it sends the data packets.

\subsection{Delay Calculation Function}

According the expectation of user, the ideal node can work following this model under which the throughput delay and so on are more important when it's energy is sufficient, but the node need to save its power to length the lifetime when its energy becomes lower.

The forwarding delay should be able to assure the stable node, whose average delay is small ,to forward packets and fight for the path, besides avoid the confliction due to concurrent communication. We call the delay calculation function Dt. It contains of two parts as the formula 1 . Tc calculated by the load aims to guarantee the forwarding priority of nodes with sufficient energy, while $\theta$ is the random value for avoiding confliction.

$$
D t=T_{c}+\theta
$$

The Dt's value is corresponding with the forwarding waiting time, the bigger the value the longer the waiting time.

\section{Simulation}

The proposed AODVLB protocol's performance is analyzed using NS2 simulator. The network is 
planned and implemented using network simulator with maximum of 120 nodes . The locations of the nodes are random and other parameters based on which the network is shaped are given in Table1. The simulator is applied with traditional AODV and proposed AODVLB, the results are obtained for assessment. The proposed AODVLB protocol has shown large progress over the Qos parameters such as average delay.

Table 1

\begin{tabular}{cccc}
\hline Parameter & Value & Parameter & Value \\
\hline Network Size & $200 \mathrm{~m}^{*} 100 \mathrm{~m}$ & Max & 10 \\
Number of & & connection & \\
nodes & $60 、 80 、 100$ & time & $400 \mathrm{~s}$ \\
Maximum & & MAC layer & \\
speed & $18 \mathrm{~km} / \mathrm{h}$ & protocol & Simple Mac \\
Transmission & & & \\
range & $60 \mathrm{~meters}$ & Initial Energy & $50 \mathrm{~J}$ \\
\hline Packet size & $512 \mathrm{~kb}$ & Tx & $1.8 \mathrm{~mW}$ \\
\hline Traffic type & CBR & Rx & $1.3 \mathrm{~mW}$ \\
Transmission & 20 times per & Route & AODVLB、 \\
rate & second & Protocols & AODV \\
\hline Titter & & &
\end{tabular}

\subsection{Jitter}

Jitter is the performance reflecting the latency of data packets in translating process. The paper define the average delay as the time difference from the data sending time to the data receiving time, apart from the route finding time, as formula 2.

$$
A D=\sum_{i=1}^{n} \frac{\operatorname{Re} \text { ceiveT }[i]-\operatorname{Send} T[i]}{n}
$$

The results are showing in table 2 . In the scene with 60 nodes the performance is as the same in AODVDF and AODV, but the AODVDF's performance is little less than AODV's in other scene. That is because the AODVDF is to select the stable path which contains more intermediate nodes than AODV and will use more time.

\begin{tabular}{|c|c|c|}
\multicolumn{3}{c}{ Table2 Jitter } \\
\hline Scene Size & AODVLB & AODV \\
\hline bonodes & 0.28 & 0.28 \\
\hline
\end{tabular}

\begin{tabular}{|c|c|c|}
\hline 8onodes & 0.46 & 0.13 \\
\hline 120nodes & 0.41 & 0.18 \\
\hline
\end{tabular}

\subsection{Network lifetime}

Network lifetime is an important indicator in performance. The relationship between the number of exhausted nodes and the running time can reflect the state of the networks. It's easy to understand, the longer the running time with the same exhausted nodes, the longer the network lifetime. The figure 1 is the simulation results under 80 nodes.

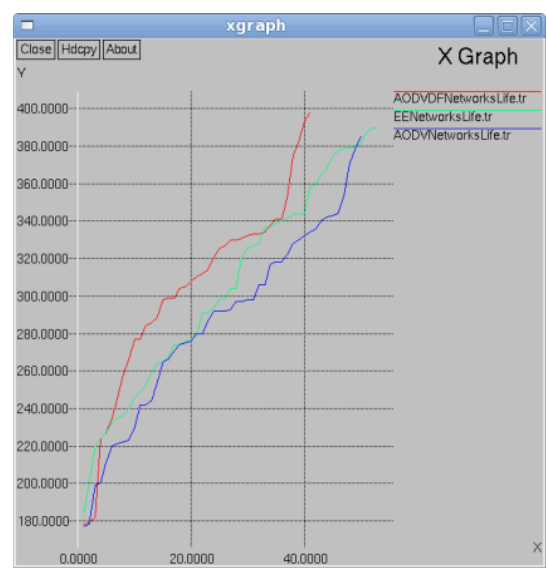

Fig.1: 80nodes

$\mathrm{X}$ axis denotes the number of exhausted nodes and $\mathrm{Y}$ axis denotes the net running time. The communicating frequency is 20 and number of link is 6.We can acknowledge that the network lifetime of AODVLB is longer than the AODV's, obviously, while the accurate value of improving is show in table 3. The traditional AODV doesn't provide reliable routing since the nodes in the network are not checked against its energy levels. While the low energy level may cause packets lost because of draining out of energy.

Table 3: The improvement on network lifetime

\begin{tabular}{|c|c|c|c|}
\hline scene & $\begin{array}{c}60 \mathrm{nod} \\
\text { es }\end{array}$ & $\begin{array}{c}80 \mathrm{nod} \\
\text { es }\end{array}$ & $\begin{array}{c}120 \mathrm{nod} \\
\text { es }\end{array}$ \\
\hline $\begin{array}{c}\text { Improvement } \\
\text { Rate }\end{array}$ & $\begin{array}{c}23.3 \\
\text { Remark: }\end{array}$ & $\% .1$ & $13.0 \%$ \\
\hline \multicolumn{3}{|c|}{ Im provementRate $=\frac{\text { AODVLB }-A O D V}{A O D V}$} \\
\hline
\end{tabular}

\subsection{Throughput Capacity}

Throughput capacity is an important 
performance metric reflecting the capability about the load carrying capacity of networks. The throughput is affected by the combined effects of different layers, radio channel confliction and node's cache. The proposed AODVDF can improve the throughput of networks, by selecting the stable path. The results is as table 4. The AODV is to choose the shortest path, but these paths may be unstable and failed soon. We can get the conclusion that the AODVDF can assume a greater load than the standard aodv protocol in sparse node scene.

Table 4 Received Packets Number

\begin{tabular}{|c|c|c|}
\hline Scene Size & AODV & AODVLB \\
\hline 60 & 1461 & 3445 \\
\hline 80 & 2101 & 2559 \\
\hline 120 & 2390 & 2630 \\
\hline
\end{tabular}

\subsection{Result}

According to the simulation results, the AODVLB is much more excellent in these metrics, balancing the average delay, extending the networks lifetime and throughput, especially in sparse nodes scene.

\section{Summary and Improvement}

The simulation results show that AODVLB is more efficient comparing with the AODV. We observed that there are load balancing and delay forwarding in AODVLB. All the nodes are used and the most optimized path is built and kept according to the current network state.

The AODVLB expands the improvement in route protocol to a new area, controlling the path building, but it is still imperfect and deficient in running path monitor, modifying failed path. Both of them should be considered.

\section{References}

[1] Sihai Zheng, Layuan Li, Yong Li. A QoS Routing Protocol for Mobile Ad Hoc Networks Based on Multipath. JOURNAL OF NETWORKS, VOL. 7, NO. 4, 2012:691-698

[2] Mina Masoudifar. A review and performance comparison of QoS multicast routing protocols for MANETs. Ad Hoc Networks. 2009(7):1150-1155

[3] Peter Jurcik. Real-time Communication over
Cluster-tree Wire-less Sensor Networks [R] . IPP-HURRAY Technical Report, Porto, Portugal, 2010.

[4] Yih-Chun Hu,(2003), Enabling Secure High-Performance Wireless Ad Hoc Networking, PhD Thesis, Carnegie Mellon University (CMU)

[5] Ilyas M.,(2003), The Handbook Of Wireless Ad Hoc Network, CRC

[6] Guoqiang Wang, Damla Turgut,Ladislau Bölöni.Improving routing performance through $\mathrm{m}$-limited forwarding in power-constrained wirless ad hoc networks.ScienceDirect. 2008(68):501-514

[7] Song JunKeun, Ryoo Jeong-dong, Kim SangCheol, et al. A Dynamic GTS Allocation Algorithm in IEEE 802.15.4 for QoS guaranteed Real-time Applications[C]// Proc of IEEE Int'l Symp on Consumer Electronics, 2007:1-6.

[8] S. Santhi,G. Sudha Sadasivam.Enhanced AOMDV for energy conservation in hybrid ad hoc networks[J]. Information and Communication Technology. 2014, Vol. 6(2):122-141.

[9] Wang Qingwen, Shi Haoshan, Qi Qian. A Cross-Layer Dynamic Probabilistic Broadcasting Strategy for Mobile Ad Hoc Networks[C]. //Proceedings of the Education Technology and Computer Science (ETCS), 2011 Third International Workshop on: vol.2: MECS, 2012:62-65.

[10] RamachandranI,Das A K,RoyS.Analysis of the contention access period of IEEE 802.15.4[J].ACM Transactions on SensorNetworks,2007,3(1) 